\section{Biotempo (Lima)}

https://revistas.urp.edu.pe/index.php/Biotempo

\section{lationdex̂ catálogo 8}

REVIEW ARTICLE / ARTÍCULO DE REVISIÓN

\author{
A MARINE ANNELID AS A TREATMENT ALTERNATIVE \\ FOR COVID-19
}

\title{
UN ANÉLIDO MARINO COMO ALTERNATIVA DE TRATAMIENTO PARA COVID-19
}

\author{
Melissa Herrera-Perez ${ }^{1, *}$
}

1 Facultad de Ciencias Biológicas. Universidad Ricardo Palma (URP). Santiago de Surco, Lima, Perú; irene.herrera@urp.edu.pe, imelissa.herrera.p@gmail.com

* Corresponding author: irene.herrera@urp.edu.pe/imelissa.herrera.p@gmail.com

\begin{abstract}
The world is facing a pandemic caused by the viral infection of the SARS coronavirus- CoV-2 that triggers the COVID-19 disease. Currently, no specific treatment or vaccine is known to counteract the symptoms and conditions caused by the virus, but antiviral, anti-inflammatory, immunomodulatory, mechanical support treatment, hematic, and others are being administered based on multi-organ dysfunction. When facing a new virus, the treatments used have been combined, which have generated secondary clinical effects in the patient. This study will focus on the hematic mechanical support treatment, where the patient is in a state of oxygen deficiency. During this phase of development of the disease that the extracellular hemoglobin extracted from Arenicola marina (Linnaeus, 1758) can be an alternative treatment through a blood transplant, as it can transport up to 40 times more oxygen when compared to hemoglobin in human. According to studies, extracellular hemoglobin has a natural antioxidant that does not induce mutagenic responses, immunogenic or allergenic, granting high affinity and cooperativity in bonding with oxygen, high structural stability, and high resistance to oxidation of the heme group, unlike human hemoglobin. The result of these promising investigations of scientifictechnological development allowed to name this new molecule, as HM-101, which has been purified and used for the production of HEMOXYCarrier (HC, Hemarina SA, France), oxygen carrier for therapeutic purposes. This document was prepared through a bibliographic search in PubMed and Google Scholar using the following words: coronavirus, COVID-19, or SARS-CoV-2, A. marina and treatment, treatment or therapy. Therefore, it is intended to inform about the technological scientific knowledge of extracellular hemoglobin extracted from A. marina as an alternative treatment for COVID-19, with promising clinical trials as a possible substitute for human hemoglobin. Likewise, the potential of scientific-technological research in different study areas with "polychaete" annelids, based on basic research in Peru.
\end{abstract}

Keywords: Arenicola marina - extracellular hemoglobin - COVID-19 - treatments 
El mundo se está enfrentando una pandemia causada por la infección viral del coronavirus SARS-CoV-2 desencadenante de la enfermedad COVID-19. Actualmente, no se conoce ningún tratamiento específico ni vacuna para contrarrestar los síntomas y afecciones causadas por el virus, pero se están administrando tratamientos: antivirales, antiinflamatorios, inmunomodulador, tratamiento de soporte mecánico, hemático y otros en función de la disfunción multiorgánica. Al enfrentarnos a un nuevo virus los tratamientos empleados han sido combinados, los cuales han generado efectos clínicos secundarios en el paciente. El presente documento se centrará en el tratamiento de soporte mecánico, hemático, donde el paciente se encuentra en estado de deficiencia de oxígeno. Durante esta fase de desarrollo de la enfermedad es donde la hemoglobina extracelular extraída de Arenicola marina (Linnaeus, 1758) puede ser una alternativa tratamiento a través de un trasplante sanguíneo pues puede transportar hasta 40 veces más oxígeno, al compararla con la hemoglobina humana. Según estudios, la hemoglobina extracelular posee un antioxidante natural no induciendo respuestas mutagénicas, inmunogénicas o alergénicas otorgando alta afinidad y cooperatividad en la unión con el oxígeno, alta estabilidad estructural y alta resistencia a la oxidación del grupo hemo a diferencia de la hemoglobina humana. El resultado de estas investigaciones promisorias de desarrollo científico tecnológico permitió denominar a esta nueva molécula, como HM-101, la cual ha sido purificada y utilizada para la producción de HEMOXYCarrier (HC, Hemarina S.A., Francia), transportador de oxígeno para fines terapéuticos. El presente documento se elaboró mediante una búsqueda bibliográfica en PubMed y Google Scholar, usando las siguientes palabras: coronavirus, COVID-19, o SARS-CoV-2, A. marina y tratamiento, treatment o therapy. Por ende, se pretende informar sobre el conocimiento científico tecnológico de la hemoglobina extracelular extraída de $A$. marina como alternativa de tratamiento para el COVID-19, siendo sus ensayos clínicos promisorios como posible sustituyente de la hemoglobina humana. Así mismo, como potencial de investigación científico tecnológico en diferentes áreas de estudio con anélidos "poliquetos", a partir de su investigación básica en el Perú.

Palabras clave: Arenicola marina - hemoglobina extracelular - COVID-19 - tratamientos

\section{INTRODUCCIÓN}

Los anélidos incluyen a las lombrices de tierra y a las sanguijuelas. Sin embargo, el grupo más abundante y rico en especies es el de los "poliquetos", donde la mayoría de sus especies son marinas (Tovar-Hernández et al., 2013).

Una de sus especies, Arenicola marina (Linnaeus, 1758), perteneciente a la Familia Arenicolidae, es una de las 87 familias que contiene la "Clase Poliqueta", que habita en la zona intermareal, y es conocido como "gusano de lodo" o "gusano de arena". Es un animal grueso con 13 pares de branquias muy ramificadas y coloridas a lo largo de parte de su cuerpo. Se caracteriza por construir madrigueras de 20 a $40 \mathrm{~cm}$ de profundidad como un tubo abierto en forma de L (Wells, 1945; Wells, 1966; Riisgård et al., 1996; Zebe \& Schiedek, 1996; Reise, 2002). Esta madriguera se genera y mantiene porque el anélido ingiere el sedimento, ayudado por el bombeo de agua a través de las contracciones rítmicas de los músculos circulares, y porque deposita sus heces en la superficie. Las madrigueras son fácilmente reconocibles en marea baja, debido a la presencia de un agujero rodeado por un montículo de sedimento, producto de la acumulación del material expulsado por el gusano (Wells, 1945; Wells, 1966; Zebe \& Schiedek, 1996). La especie se considera alimentador de depósito no selectivo; ingiere constante cantidad de sedimento mediante una faringe especializada y al hacerlo, funciona como remediador al metabolizar sedimento con alto contenido de materia orgánica, metales pesados, agrotóxicos, xenobióticos, entre otros (Longbottom, 1970; Riisgård et al., 1996; Rasmussen et al., 2020; González-Zuarth \& Vallarino, 2014; De Cubber et al., 2020).

Arenicola marina, es un oxiconformador, es decir que su consumo de oxígeno dependerá de la disponibilidad del mismo en su ambiente, permitiéndole sobrevivir durante la marea baja o durante periodos prolongados de tiempo (hasta 20 días) sin presencia de oxígeno, al cambiar su metabolismo aeróbico a anaeróbico, disminuye su tasa metabólica. Cuando esto sucede, la hemoglobina extracelular de Arenicola se satura, así como mediante la mezcla de sangre oxigenada y no oxigenada, favoreciendo su afinidad con el oxígeno, continuando su transporte y liberación a los diferentes tejidos. 
La hemoglobina extracelular es un tipo de pigmento respiratorio, denominado así por no encontrarse dentro de una célula circulando en la sangre a diferencia de la hemoglobina humana, el cual se encuentra dentro del glóbulo rojo (Longbottom, 1970; Toulmond, 1975; Zebe \& Schiedek, 1996; Glasby et al., 2000).

El estudio de este pigmento se inició desde 1912 por el científico Peyrega, quién observó una similitud en el espectro de absorción del pigmento respiratorio con la oxihemoglobina de la sangre de caballo (mamífero), informando su aislamiento mediante la técnica de cristales de hemina (técnica forense para determinar presencia de sangre en superficie). El interés de investigación por su estructura, se inició a partir de 1960, 30 años después de la determinación de su peso molecular. Las primeras observaciones se iniciaron utilizando el microscopio electrónico de transmisión y la primera micrografía de esta molécula fue obtenida de A. marina. Esta imagen revela una estructura hexagonal con diámetros de 22$26 \mathrm{~nm}$, donde cada molécula estaba constituida de dos hexágonos superpuestos con una altura de 11-17 nm (Patel \& Spencer, 1963; Zal et al., 1997).

Las diversas investigaciones básicas sobre la hemoglobina extracelular ha permitido realizar investigaciones aplicadas determinando su potencial uso como posible sustituyente de la hemoglobina humana (Rousselot et al., 2006a; Jernshøj et al., 2013), siendo una alternativa de tratamiento para la enfermedad COVID-19.

Los síntomas ocasionados por la infección viral de SARS-CoV-2, virus que desencadena la enfermedad COVID-19, puede ser desde leve caracterizada por una gripe "común", hasta severa ocasionando en el paciente el síndrome de dificultad respiratoria aguda (SDRA), sepsis, shock séptico y fallo multiorgánico finalizando en su fallecimiento. Actualmente, no existe ningún tratamiento específico ni vacuna contra el SARSCoV-2, pero se están utilizando diferentes tratamientos para combatirlo, aunque no hay evidencia científica suficiente que la enfermedad (COVID19) es progresiva, desarrollándose en tres fases (Díaz et al., 2020), siendo en cada fase un tratamiento diferente para el paciente (dependerá del historial clínico del mismo). La fase inicial se caracteriza por una elevada carga vírica, por lo cual se le administra un tratamiento antivírico con lopinavir/ritonavir, hidroxicloroquina, cloroquina (estos dos no son antivirales pero son los más usados en España, forman parte del tratamiento preventivo de la malaria), interferón-1b, azitromicina, umifenovir, remdesivir, favipiravir, siendo la mayoría de sus estudios en infecciones por otros virus (Díaz et al., 2020; Estella \&
Garnacho-Montero, 2020; Islam, 2020; Jamshaid et al., 2020; Palacios et al., 2020; Xu et al., 2020), incluso terapia con plasma de personas convalecientes (Xu et al., 2020). En la segunda fase, predomina la respuesta inflamatoria (ocasionado generalmente por la tormenta de citoquinas), utilizando el fármaco corticoides; mientras que para un tratamiento inmunomodulador se utiliza tocilizumalab (interleucina-6), aunque sólo se han realizado 2 estudios retrospectivos, tratamiento con células madres mesenquimales, entre otros. Finalmente, la última fase cuando el paciente se encuentra en estado crítico, donde se desencadena dificultad para respirar, sepsis, shock séptico y trombosis, ocasionando generalmente el fallecimiento del paciente. El tratamiento utilizado durante esta fase es soporte mecánico: intubación orotraqueal (IOT) precoz, secuencia de IOT con inducción rápida, sin ventilación con balón resucitador, maniobra de decúbito prono durante las primeras $24 \mathrm{~h}$, ventilación mecánica no invasiva (VNI), Oxigenoterapia de Alto Flujo (OAF); hemático con heparina de bajo peso molecular y otros en función de la disfunción multiorgánica (Ballesteros-Sanz et al., 2020; Cinesi et al., 2020; Estella \& Garnacho-Montero, 2020; Hernández-Garcés et al., 2020; González-Castro et al., 2020; Xu et al., 2020). La administración combinada de estos medicamentos han desencadenado efectos sinérgicos desencadenando en el paciente otras implicaciones clínicas (Ballesteros-Sanz et al., 2020; Díaz et al., 2020; Estella \& GarnachoMontero, 2020; Patrì \& Fabbrocini, 2020).

De los diferentes tratamientos el presente documento se centra en la comparación de los tratamientos utilizados cuando el paciente se encuentre en un cuadro respiratorio agudo (SDRA), trombosis, neumonía por deficiencia de oxígeno. Entonces, ¿cómo la hemoglobina extracelular puede ser una alternativa de tratamiento para el COVID-19?.

\section{MATERIALES Y MÉTODOS}

Para la elaboración de este documento se ha realizado una búsqueda bibliográfica en PubMed para literatura biomédica de MEDLINE, revistas de ciencias de la vida, y libros online y Google Scholar con las palabras coronavirus, COVID-19, o SARS-CoV-2, A. marina y tratamiento, treatment o therapy.

\section{RESULTADOS Y DISCUSIÓN}

Para saberlo, es importante explicar el proceso de infección del virus (SARS-CoV-2). Cuando los seres 
humanos inhalamos, ingresamos aire a nuestros pulmones, el cual está compuesto de diferentes gases, siendo el oxígeno, uno de los más representativos. El oxígeno es conducido mediante las vías respiratorias hacia el pulmón dirigiéndose hasta los alvéolos, donde por difusión (alvéolo-capilar) ingresa al torrente sanguíneo mediante los capilares, siendo intercambiado por el dióxido de carbono liberado en la expiración. El oxígeno ingresado al torrente sanguíneo, se dirige mediante la vena pulmonar al corazón, para nuevamente salir y dirigirse a los demás órganos de nuestro cuerpo y así suministrar oxígeno para cumplir nuestras diferentes funciones. Entonces, cuando el virus ingresa por la vía respiratoria se aloja en los alvéolos de los pulmones, es ahí cuando nuestro sistema inmunitario innato actúa rápidamente iniciando el proceso de reconocimiento celular mediante receptores específicos, reconociendo a los agentes externos como cuerpo extraño, e iniciando el mecanismo de defensa, activando y liberando simultáneamente células y moléculas requeridas para su eliminación, como macrófagos, neutrófilos, el sistema del complemento, activando a la vez, citoquinas, las cuales estimulan la inflamación en los alvéolos y posteriormente al pulmón, con la finalidad de proteger al organismo, que por medio de vasodilatación e incremento de la permeabilidad capilar permite la llegada de más células y moléculas al lugar de infección aumentando su número de forma exorbitante, denominado comúnmente como "tormenta de citoquinas" (De Pablo-Sánchez et al., 2005; Reyes \& García, 2005; Carrasco, 2011; Librado, 2011).

El número de células de defensa es tan numeroso que el proceso encargado de su regulación, no puede contrarrestarlo, ocasionando daño en los tejidos y órganos, desencadenando, producto de la inflamación (sepsis), neumonía, y hemorragia interna, debido a la permeabilidad de los capilares la sangre se libera de su vía de transporte, a su vez, se activa el proceso de coagulación, originando una coagulación intravascular diseminada (CID), derivándose a trombosis, ocasionando un daño multiorgánico, por la incapacidad de transportar oxígeno donde finalmente el paciente muere (Durval et al., 2006; Páramo, 2006; de Juan Bagudá et al., 2020; Driggin et al., 2020; Mendoza et al., 2020; Perez-Girbes, 2020; Vivas et al., 2020). Es durante esta fase crítica de la enfermedad donde el trasplante de sangre con hemoglobina extracelular $(\mathrm{AmHb})$ proveniente de A. marina sería un tratamiento alternativo. Este pigmento respiratorio natural tiene alto peso molecular $(-3600 \mathrm{kDa})(\mathrm{HBL}$ $\mathrm{Hbs})$, y puede contrarrestar la dificultad del transporte de oxígeno de la hemoglobina humana, al transportar hasta 40 veces más oxígeno al encontrarse en disolución. Esta molécula gigante de estructura cuaternaria, constituida por una bicamada hexagonal (HBL Hbs), ordenada mediante dos hexágonos superpuestos, constituida por 156 globinas (Cada molécula de globina tiene un grupo hemo (ion $\mathrm{Fe}$ ) y 44 unidades que no presentan el grupo prostético), transportando hasta f156 moléculas de oxígeno cuando está saturado (Waxman, 1971; Pionetti \& Pouyet, 1980; Vinogradov et al., 1980; Zal et al., 1997; Chabasse et al., 2006a, 2006b; Rousselot et al., 2006ab; Bailly et al., 2007; Moreira et al., 2011; Jernshøj et al., 2013; Zal \& Rousselot, 2014; Marchand et al., 2017). Posee un antioxidante natural de propiedad similar a la superóxido dismutasa, la cual no induce respuestas mutagénicas, inmunogénicas o alergénicas y se degrada en cadenas polipeptídicas y hemo (Moon-Massat et al., 2015, Le Meur et al., 2020). Estas características, son las que le otorga alta afinidad y cooperatividad en la unión con el oxígeno (P50 7 mm Hg) (Moon-Massat et al., 2017), alta estabilidad estructural y alta resistencia a la oxidación del grupo hemo (ion Fe) (Rousselot et al., 2006a, 2006b; Zal \& Rousselot, 2014).

Le Gall et al. (2014), Le Meur et al. (2010), Lemaire et al. (2019), Rousselot et al. (2006a), (2006b), Moon-Massat (2017) y Tsai et al. (2012) informan que la hemoglobina extracelular de $A$. marina es una molécula gigante denominada Hemarina-M101 que ha sido purificada y utilizada para la producción de HEMOXYCarrier (HC, Hemarina S.A., France), producto desarrollado como transportador de oxígeno para fines terapéuticos, que en disolución puede alcanzar y entregar oxígeno a diferentes tejidos inaccesible por los glóbulos rojos. Opera en un simple gradiente de presión parcial de oxígeno, permitiendo que la absorción de oxígeno y su liberación no requiere de un efector alostérico. Esta proteína no desencadena respuestas inmunológicas, alegrías o efectos protrombóticos, así como ningún efecto en la vasoconstricción o daño al corazón. La eficacia de HM-101 usado en ex vivo como aditivo para soluciones de conservación para prevenir lesiones por isquemia, reperfusión se ha demostrado en estudios preclínicos en riñón, corazón y pulmón, como lo demuestran Lemaire et al. (2019), en la preservación de rata y humano y Le Meur et al. (2020), en el primer trasplante renal en humano, aunque no se conocen investigaciones que se han realizado en pacientes con COVID-19, HM101 como transportador de oxígeno sería una nueva y favorable alternativa como tratamiento para pacientes que se encuentren con insuficiencia respiratorio aguda, ya que para ello se utilizan mecanismos de tratamiento tradicionales, siendo un factor de riesgo de contagio para los pacientes y el personal de salud por su alta transmisibilidad y dispersión de partículas 
virales requiriendo el uso de filtros adecuados para su procedimiento (Hernández-Garcés, et al., 2020, Yago et al., 2020).

Si bien en las costas del Perú se identificó $A$. marina por Cabanillas et al. (2016) es fundamental verificar su identificación taxonómica, e iniciar estudios a nivel básico para conocer, comprender, desarrollar e iniciar investigaciones aplicadas, pues $A$. marina está identificado para la costa del Mar del Norte (mar marginal del océano Atlántico, situado al este, entre las costas de Noruega y Dinamarca, al oeste de las islas británicas y las de Alemania, los Países Bajos, Bélgica y al sur de Francia), indicando que la especie identificada en nuestro país sea una nueva especie; así como otras especies potenciales. Esta confusión es frecuente en los poliquetos al ser considerados muchos de ellos como cosmopolita, animales con una amplia distribución geográfica (Hutchings \& Kupriyanova, 2018).

Así mismo, el estudio sobre "poliquetos" en el Perú es escaso, porque los especialistas dedicados a la taxonomía de poliquetos son pocos, de los cuales ninguno se dedica exclusivamente a la investigación, haciendo los avances más lentos. Igualmente, el avance en conocimiento e identificación de nuestras especies a nivel taxonómico debe estar acompañado con la elaboración de una guía taxonómica; y el desarrollo de una colección científica como menciona Aguirre \& Canales (2009), la cual se encuentre interconectada entre instituciones públicas y privadas que desarrollen investigación básica y aplicada, como ha sido desarrollado por países vecinos, fundamental para desenvolver investigaciones a nivel tecnológico permitiendo no depender de tecnologías extranjeras.

En la actualidad los estudios a nivel mundial con poliquetos abarca diferentes áreas de investigación como en ecología, siendo varias especies de ellas bioindicadores de diversidad, ecológicos y ambientales informando cualitativamente el estado actual de un ecosistema; estudios aplicados a nivel ecotoxicológico mediante la realización de bioensayos de toxicidad, permitiendo también conocer el estado actual del ecosistema, así como su protección ante cualquier perturbación ambiental, o en el desarrollo de biomarcadores observando cambios genéticos, bioquímicos, moleculares, celulares, fisiológicos y morfológicos al exponerlos frente a un estresor o sustancia xenobiótica, siendo estudios cuantitativos (González-Zuarth \& Vallarino, 2014). Solo en Perú se ha realizado un estudio para conocer las condiciones óptimas de temperatura y salinidad para el cultivo de Capitella sp. con la finalidad de realizar estudios reproductivos para su utilización como especies de prueba en estudios ecológicos, ecotoxicológicos, biotecnológicos, alimenticios y reproductivos por Herrera-Perez \& Méndez (2019ab). Así mismo, estudios en neurobiología, como modelos biológicos para entender cómo se desarrollan enfermedades degenerativas como el Alzheimer o Parkinson; en inmunología, farmacología, biología del desarrollo, biología reproductiva, biología molecular y bioquímica. Incluso, como alternativa de alimento para el cultivo de recursos hidrobiológicos al presentar altos niveles de ácidos grasos, proteínas y aminoácidos (Poltana et al., 2007; Palmer et al., 2014; Pombo et al., 2018), abriendo a su vez, otras áreas de investigación a nivel tecnológico, enfatizando nuevamente que no se puede avanzar en estas áreas sino sabemos qué especies nos representan.

\section{CONCLUSIÓN}

El presente manuscrito propone informar sobre el conocimiento de un tratamiento alternativo para el COVID-19 producto del desarrollo científico tecnológico de la hemoglobina extracelular de $A$. marina, siendo una nueva alternativa como tratamiento para pacientes que se encuentran con insuficiencia respiratoria aguda, a partir de un trasplante sanguíneo al no desencadenar respuestas inmunológicas, alergias o efectos protrombóticos y al presentar alta afinidad y cooperatividad en la unión con el oxígeno cuando se encuentra en disolución a diferencia de la hemoglobina humana. Además, del potencial e importancia en investigación básica en diferentes áreas de estudio sobre anélidos "poliquetos" en el Perú, fundamental para desarrollar investigación aplicada.

\section{AGRADECIMIENTOS}

La autora agradece a: Paulo Lana, Sergio Salazar, Ricardo Castro y Luis Aguirre por sus sugerencias, comentarios y motivación para la realización del presente artículo.

\section{REFERENCIAS BIBLIOGRÁFICAS}

Aguirre, L. \& Canales, R. 2009. Poliquetos de Perú: estado actual y perspectivas para la investigación. En: Díaz-Díaz, O.; Bone, D.; Rodríguez, C.T. \& Delgado-Blas, V.H. (Eds.) Poliquetos de Sudamérica. Cumaná, Venezuela. Volumen especial del Boletín del Instituto Oceanográfico de Venezuela, pp.101-114. 
Bailly, X.; Chabasse, C.; Hourdez, S.; Dewilde, S.; Martial, S.; Moens, L. \& Zal, F. 2007. Globin gene family evolution and functional diversification in annelids. Federation of European Biochemical Societies, 274: 2641-2652.

Ballesteros-Sanz, M.Á.; Hernández-Tejedor, A.; Estella, Á.; Jiménez, R.J.; González de Molina, O.F.J.; Sandiumenge Camps, A.; Vidal, C.P.; de Haro, C.; Aguilar, A.E.; Bordejé, L.L.; García, S.I.; Bodí, M.; García, S.M.; Párraga, R.M.J.; Alcaraz, P.R.M.; Amézaga, M.R.; Burgueño, L.P. Grupos de Trabajo de la Sociedad Española de Medicina Intensiva, Crítica y Unidades Coronarias (SEMICYUC), Grupos de Trabajo de la Sociedad Española de Medicina Intensiva, Crítica y Unidades Coronarias. Grupo de Trabajo de Bioética, Grupo de Trabajo de Cuidados Intensivos Cardiológicos y RCP, Junta Directiva. 2020. Recomendaciones de «hacer» y «no hacer» en el tratamiento de los pacientes críticos ante la pandemia por coronavirus causante de COVID-19 de los Grupos de Trabajo de la Sociedad Española de Medicina Intensiva, Crítica y Unidades Coronarias (SEMICYUC). Medicina intensiva, 44: 371-388.

Cabanillas, R.; Advíncula, O. \& Gutiérrez, C. 2016. Diversidad de Polychaeta (Annelida) en el intermareal de los esteros del Santuario Nacional los Manglares de Tumbes, Perú. Revista Peruana de Biología, 23: 117-126.

Carrasco, O.L. 2011. Citoquinas: De fieles aliadas a temibles enemigas. Real Academia de Ciencias Veterinarias de Andalucía Oriental, Anales, 24: 75-90.

Chabasse, C.; Bailly, X.; Sanchez, S.; Rousselot, M. \& Zal, F. 2006a. Gene structure and molecular phylogeny of the linker chains from the giant annelid hexagonal bilayer Hemoglobins, Journal of Molecular Evolution, 63: 365-374.

Chabasse, C.; Bailly, X.; Rousselot, M. \& Zal, F. 2006 b. The multigenic family of the extracellular hemoglobin from the annelid polychaete Arenicola marina. Comparative Biochemistry and Physiology, 144: 319-325.

Chadwick, R.A.; Barrow, M. \& Wells, R.M.G. 1984. Aspects of respiratory function in the blood of the intertidal lugworm Abarenicola affinis (Polychaeta), New Zealand. Journal of Marine and Freshwater Research, 18: 171-175.
Cinesi, G.C.; Peñuelas, R.Ó.; Luján, T.M.l.; Egea, S.C.; Masa, J.J.F.; García, F.J.; Carratalá-Perales, J.M.; Heili-Frades, S.B.; Ferrer-Monreal, M.; Nilsson, M.A.; Lista-Arias, E.; Sánchez-Rocamora, J.L.; Garrote, J.I.; Zamorano-Serrano, M.J.; González-Martínez, M.; Farrero-Muñoz, E.; Mediano-San Andrés, O.; Cervera, G.R.; MasSerra, A.; Hernández-Martínez, G.; HaroLópez, C.H.; Roca-Gas, O.; Ferrer-Roca, R.; Romero-Berrocal, A. \& Ortola, C.F. 2020. Recomendaciones de consenso respecto al soporte respiratorio no invasivo en el paciente adulto con insuficiencia respiratoria aguda secundaria a infección por SARS-CoV-2. Revista Española de Anestesiología y Reanimación, 67: 261-270

De Cubber, L.; Lefebvre, S.; Lancelot, T.; Duong, G. \& Gaudron, S.M. 2020. Investigating down-shore migration effects on individual growth and reproduction of the ecosystem engineer Arenicola marina. Journal of Marine Systems, doi: https:// doi.org/10.1016/ j.jmarsys.2020.103420

de Juan Bagudá, J.; Farrero, T.M.; García-Cosío, M.D.; Recio, M.A.; Sabé, F.N.; García, P.J.M. \& González, C.J. 2020. Implicaciones de la pandemia por COVID-19 para el paciente con insuficiencia cardiaca, trasplante cardiaco y asistencia ventricular. Recomendaciones de la Asociación de Insuficiencia Cardiaca de la Sociedad Española de Cardiología. REC CardioClinics, doi. https://doi.org/10.1016/j. rccl.2020.03.007

De Pablo-Sánchez, R.; Monserrat, S.J.; Prieto, M.A.; Reyes, M.E.; Álvarez de Mon Soto, M. \& García, S.M. 2005. Balance entre citocinas pro y antiinflamatorias en estados sépticos. Medicina Intensiva, 29: 151-158.

Díaz, E.; Amézaga, M.R.; Vidal, C.P.; Escapa, M.G.; Suberviola, B.; Serrano, L.A.; Marcos, N.P.; Quintana, D.M. \& Catalán, G.M. 2020. Tratamiento farmacológico de la COVID-19: revisión narrativa de los Grupos de Trabajo de Enfermedades Infecciosas y Sepsis (GTEIS) y del Grupo de Trabajo de Transfusiones Hemoderivados (GTTH). Medicina Intensiva, doi: https://doi.org/10.1016/j. medin.2020.06.017

Driggin, E.; Madhavan, M.V.; Bikdeli, B.; Chuich, T.; Laracy, J.; Bondi-Zoccai, G.; Brown, T.S.; 
Nigoghossian, C.D.; Zidar, D.A.; Haythe, J.; Brodie, D.; Beckman, J.A.; Kirtane, A.J.; Stone, G.W.; Krumholz, H.M. \& Parikh, S.A. 2020. Cardiovascular considerations for patients, health care workers, and health systems during the Coronavirus Disease 2019 (COVID-19) Pandemic. Journal of the American College of Cardiology, doi: https://doi.org/10.1016/j. jacc.2020.03.031

Durval, C.K.; Calasans, M.T.A. \& Leal, M.C.V. 2006. Citocinas pró-inflamatórias e dor. Revista Brasileira de Reumatologia, 46: 199-206.

Estella, Á. \& Garnacho-Montero, J. 2020. Del empirismo a la evidencia científica en el tratamiento con antivíricos en los casos graves de infección por coronavirus en tiempos de epidemia. Medicina Intensiva, 2020. doi: https://doi.org/10.1016/j. medin.2020.04.009.

Glasby, C.J.; Hutchings, P.A.; Fauchald, K.; Paxton, H.; Rouse, G.W.; Watson Russell, C. \& Wilson, R.S. 2000. Class Polychaeta. In: Beesley, P.L.; Ross, G.B. \& Glasby, C.J. (eds). Fauna of Australia. Vol. 4A. Polychaetes \& Allies: The Southern Synthesis. [Book]. pp. 1-296.

González-Castro, A.; Fajardo-Campoverde, A.; Medina, A. \& Alapontd, V.M. 2020. Ventilación mecánica no invasiva y oxigenoterapia de alto flujo en la pandemia COVID-19: El valor de un empate. Medicina Intensiva, doi: https://doi. org/10.1016/j.medin.2020.04.017

González-Zuarth, C. \& Vallarino, A. 2014. Los bioindicadores ¿una alternativa real para la protección del medio ambiente?. En: Bioindicadores: guardianes de nuestro futuro ambiental. González, Z.C.A.; Vallarino, A.; Perez, J.J.C. \& Low, P.A.M. (eds). San Cristóbal de Las Casas, Chiapas, México: El Colegio de la Frontera. pp. 21-37.

Hernández-Garcés, H., Belenguer, M.A. \& Zaragoza, C.R. 2020. Noninvasive mechanical ventilation and COVID-19. Minimizing dispersion. Ventilación mecánica no invasiva y COVID-19. Minimizando la dispersión. Medicina intensiva, doi: https://doi.org/10.1016/j. medin.2020.03.015

Herrera-Perez, M. \& Méndez, N. 2019a. Efecto de la temperatura y salinidad en la mortalidad de adultos de Capitella sp. (Polychaeta: Capitellidae) en el laboratorio. Revista de Biología Tropical, 67(S5): 51-62.

Herrera-Perez, M. \& Méndez, N. 2019b. Efecto del cadmio sobre la mortalidad de adultos de Polydora sp. (Polychaeta: Spionidae) en el laboratorio. Revista de Biología Tropical, 67(S5): 110-118.

Hutchings, P., \& Kupriyanova, E. 2018. Cosmopolitan polychaetes - fact or fiction? Personal and historical perspectives. Invertebrate Systematics, 32: $1-9$.

Islam, R.M.I. 2020. Current drugs with potential for treatment of COVID-19: A Literature Review. Journal of Pharmacy and Pharmaceutical Sciences, 23: 58-64.

Jamshaid, H.; Zahid, F.; Din, I.U.; Zeb, A.; Choi, H.G.; Khan, G.M. \& ud-Din, F. 2020. Diagnostic and treatment strategies for COVID-19. American Association of Pharmaceutical Scientists PharmSciTech, 21: 222.

Jernshøj, K.D.; Hassing, S. \& Olsen, L.F. 2013. A combination of dynamic light scattering and polarized resonance Raman scattering applied in the study of Arenicola marina extracellular hemoglobin. The Journal of Chemical Physics, 139, doi: http://dx.doi.org/10.1063/1.4813920

Le Gall, T.; Polard, V.; Rousselot, M.; Lotte, A.; Raouane, M.; Lehna, P.; Opolone, P.; Leizef, E.; Deutschd, E.; Zal, F. \& Montier, T. 2014. In vivo biodistribution and oxygenation potential of a new generation of oxygen carrier. Journal of Biotechnology, 187: 1-9.

Le Meur, Y.; Badet, L.; Essig, M.; Thierry, A.; Büchler, M.; Drouin, S.; Deruelle, C.; Morelon, E.; Pesteil, F.; Delpech, P.O.; Boutin, J.M.; Renard, F. \& Barrou, B. 2020. First-in-human use of a marine oxygen carrier (M101) for organ preservation: A safety and proof-of-principle study. American Journal Transplantation, 20: 1729-1738

Lemaire, F.; Sigrist, S.; Delpy, E.; Cherfan, J.; Peronet, C.; Zal, F.; Bouzakri, K.; Pinget, M. \& Maillard, E. 2019. Beneficial effects of the novel marine oxygen carrier M101 during cold preservation of rat and human pancreas. Journal of Cellular and Molecular Medicine, 23: 8025-8034. 
Librado, C.O. 2011. Citoquinas: De fieles aliadas a temibles enemigas. Anales Real Academia de Ciencias Veterinarias de Andalucía Oriental, 24: 75-90.

Longbottom, M.R. 1970. The Distribution of Arenicola Marina (L.) with particular reference to the effects of particle size and organics matter of the sediments. Journal of Experimental Marine Biology and Ecology, 5: 138-157

Marchand, A.; Crepin, N.; Roulland, I.; Semence, F.; Domergue, V.; Zal, F., Polard, V. \& Coquerel, A. 2017. Application of HBOCs electrophoretic method to detect a new blood substitute derived from the giant extracellular hemoglobin of lugworm. Drug Testing and Analysis, 9: 17621767.

Mendoza, F.F.J.; García, B.L. \& Bastarrika, G. 2020. Extensión y cuantificación de la carga inflamatoria en COVID-19 mediante tomografía computarizada. Archivos de Bronconeumología, doi: https://doi. org/10.1016/j.arbres.2020.03.020

Moon-Massat, P.; Mullah, S.H.; Abutarboush, R.; Saha, B.K.; Pappas, G.; Haque, A.; Auker, C.; McCarron, R.M.; Arnaud, F. \& Scultetus, A. 2017. Cerebral Vasoactivity and oxygenation with oxygen carrier M101 in rats. Journal of Neurotrauma, 34: 2812-2822.

Moreira, L.M.; Guaranho de Moraes, P.C. \& Furtado, M.J.R. 2011. Hemoglobina extracelular gigante de Glossoscolex paulistus: Um extraordinário sistema supramolecular hemoproteico. Química Nova, 34: 119-130.

Palacios, C.M.; Santos, E.; Velázquez, C.M.A. \& León, J.M. 2020. COVID-19, una emergencia de salud pública mundial. Revista Clínica Española, doi: https://doi.org/10.1016/j.rce.2020.03.001

Palmer, P.J.; Wang, S.; Houlinhan, A. \& Brock, I. 2014. Nutritional status of a nereidid polychaete cultured in sand filters of mariculture wastewater. Aquaculture Nutrition, 20: 675-691.

Páramo, J.A. 2006. Coagulación Intravascular Diseminada. Medicina Clínica Barcelona, 127: 785-789.

Patel, S. \& Spencer, C.P. 1963. Studies on the haemoglobin of Arenicola marina. Comparative Biochemistry and Physiology, 8: 65-82.
Patrì, A \& Fabbrocini, G. 2020. Hydroxychloroquine and ivermectin: A synergistic combination for COVID-19 chemoprophylaxis and treatment?. Journal of the American Academy of Dermatology, 82: e221.

Perez-Girbes, A. 2020. Tromboembolia pulmonar aguda y enfermedad por coronavirus (COVID-19): ¿una asociación frecuente en pacientes graves? Archivos de Bronconeumología. doi: https:// doi.org/10.1016/j.arbres.2020.04.003

Pionetti, J.M. \& Pouyet, J. 1980. Molecular Architecture of Annelid Erythrocruorins Extracellular Hemoglobin of Arenicola marina (Polychaeta). European Journal of Biochemistry, 105: 131138.

Poltana, P.; Lerkitkul, T.; Pomgtippatee-Ta, W.P.; Asuvapongpattana, S.; Wongprasert, K.; Sriurairatana, S.; Chavadej, J.; Sobhon, P.; Olive, P.J.W. \& Withyachumnarnkul, B. 2007. Culture and development of the polychaete Perinereis cf. nuntia. Invertebrate Reproduction and Development, 50: 13-20.

Pombo A.; Baptista, T.; Granada, L.; Ferreira, S.M.F.; Gonsalves, S.C.; Anjos, C.; Sá, E.; Chainho.; Cancela da Fonseca, L.; Fidalgo e Costa, P. \& Costa, J.L. 2018. Insight into aquaculture's potential of marine annelid worms and ecological concerns: a review. Reviews in Aquaculture, 12: 107-121.

Rasmussen, A.D.; Thomas, B.G. \& Andersen, O. 2000. Cadmium dynamics in estuarine sediments: effects of salinity and lugworm bioturbation. Environmental Toxicology and Chemistry, 19: 380-386.

Reise, K. 2002. Sediment mediated species interactions in coastal waters. Journal of Sea Research, 48: 127-141.

Reyes, G.M.G. \& García, T.F. 2005. Citoquinas, Inflamación y Conducta. Vertientes. Revista Especializada en Ciencias de la Salud, 8: 4-13.

Riisgård, H.; Berntsen, I. \& Tarp, B. 1996. The lugworm (Arenicola marina) pump: Characteristics, modelling and energy cost. Marine Ecology Progress Series, 138: 149-156.

Rousselot, M.; Delpy, E.; Drieu La Rochelle, C.; Lagente, V.; Pirow, R.; Rees, J.-F.; Hagege, A.; 
Le Guen, D. \& Hourdez, S. \& Zal, F. $2006 a$. Arenicola marina extracellular hemoglobin: a new promising blood substitute. Biotechnology Journal, 1: 333-345.

Rousselot, M.; Le Guen, D. \& Zal, F. 2006b. Novel dissociation mechanism of a polychaetous annelid extracellular haemoglobin. Federation of European Biochemical Societies journal, 273: 1582-1596.

Torreiro, M.A. 2018. Revisión bibliográfica: alternativas médicas a la sangre natural. (Tesis de graduación). Universidade da Coruña, España.

Tovar-Hernández, M.A.; Salazar-Silva, P.; León-González, A.; Carrera-Parra, L.F. \& Salazar-Vallejo, S.I. 2013. Biodiversidad de Polychaeta (Annelida) en México. Revista Mexicana de Biodiversidad, doi: $10.7550 / \mathrm{rmb} .32625$

Toulmond, A. 1975. Blood oxygen transport and metabolism of the confined lugworm Arenicola marina (L.). Journal of Experimental Biology, 63: 647-660.

Tsai, A.G.; Intaglietta, M.; Sakai, H.; Delpy, E.; Drieu La Rochelle, C.; Rousselot, M. \& Zal, F. 2012. Microcirculation and NO-CO studies of a natural extracellular hemoglobin developed for an oxygen therapeutic carrier. Current Drug Discovery Technologies, 9: 166-172.

Vinogradov, S.D.; Kosinski, T.F. \& Kapp, O.H. 1980. Subunits of the extracellular hemoglobin of Arenicola Marina. Biochimica et Biophysica Acta, 621: 315-323.

Vivas, D.; Roldán, V.; Esteve-Pastor, M.A.; Roldán, I.; Tello-Montoliu, A.; Ruiz-Nodar, J.M.; Cosín-Sales, J.; María Gámez, J.; Consuegra, L.; Luis Ferreiro, J.; Marín, F.; Arrarte, V.; Anguita, M.; Cequier, Á. \& Pérez-Villacastín, J. 2020. Recomendaciones sobre el tratamiento antitrombótico durante la pandemia COVID-19. Posicionamiento del Grupo de Trabajo de Trombosis Cardiovascular de la Sociedad Española de Cardiología. Revista Espańola de Cardiología, doi: https://doi. org/10.1016/j.recesp.2020.04.006
Waxman, L. 1971. The Hemoglobin of Arenicola cristata. The Journal of Biological Chemistry, 246: 73187327.

Wells, G. 1945. The Mode of Life of Arenicola marina L. Journal of the Marine Biological Association of the United Kingdom, 26: 170-207.

Wells, G. 1966. The lugworm (Arenicola) - A study in adaptation. Netherlands Journal of Sea Research, 3: 294-313.

Yago, M.; Mayayo, I.A.; López, R.G.; Parias A.M.N.; Miranda, A.P.; Aracil, M.C.; Fuentes, E.C.; Núñez, A.R.; Martínez, I.M.; Cid, J.L.; Sarrato, G.Z.; Macías, C.C. \& Hernández-Tejedor, A. 2020. Recomendaciones sobre reanimación cardiopulmonar en pacientes con sospecha o infección confirmada por SARS-CoV-2 (COVID-19). Resumen ejecutivo. Medicina intensiva, doi: https://doi.org/10.1016/j. medin.2020.05.004

Zal, F. \& Rousselot, M. 2014. Extracellular hemoglobins from annelids, and their potential use in biotechnology. Outstanding Marine Molecules, 16: 361-376.

Zal, F.; Green, B.N.; Lallier F.H.; Vinogradov, S.N. \& Toulmond, A. 1997. Quaternary structure of the extracellular haemoglobin of the lugworm Arenicola marina: a multi-angle-laser-lightscattering and electrospray-ionisation-massspectrometry analysis. European journal of biochemistry, 243: 85-92.

Zebe, E. \& Schiedek, D. 1996. The lugworm Arenicola marina: A model of physiological adaptation to life in intertidal sediments. Helgolander Meeresunters, 50: 37-68.

Xu, X.; Ong, Y. K. \& Wang, Y. 2020. Role of adjunctive treatment strategies in COVID-19 and a review of international and national clinical guidelines. Military Medical Research, 7: 22.

Received August 17, 2020. Accepted September 20, 2020. 